\title{
Classification of Desired Motion Force Based On Cerebral Hemoglobin Information*
}

\author{
Yan-Xiang Sui ${ }^{1}$, Chun-Guang Li ${ }^{2}$, Hong-Miao Zhang ${ }^{3}$, Juan $\mathrm{Li}^{4}$ \\ ${ }^{1}$ School of Mechanical and Electric Engineering, Soochow University, Suzhou, China \\ ${ }^{2}$ School of Mechanical and Electric Engineering, Soochow University, Suzhou, China \\ ${ }^{3}$ School of Mechanical and Electric Engineering, Soochow University, Suzhou, China \\ ${ }^{4}$ School of Mechanical and Electric Engineering, Soochow University, Suzhou, China

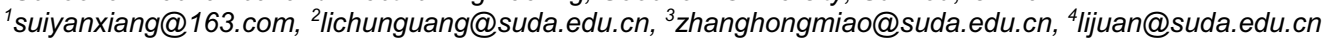

\begin{abstract}
Strength training using patients' desired force level is helpful to improve training effect and promote rehabilitation. Generally, force levels are recognized by applying EMG or biomechanical information, these methods were not suitable for patients who lost important muscle groups or have weakened muscle functions. This paper proposed a method for identifying force level based on cerebral hemoglobin information, rather than the information depending on limbs. Ten subjects performed pedaling movement in three force levels. Features were extracted in both the time-domain and frequency-domain, with deoxygenated hemoglobin (deoxy) and the difference between oxygenated hemoglobin (oxy) and deoxy as parameters. Important frequency bands $(0.01-0.03 \mathrm{~Hz}, 0.03-0.06 \mathrm{~Hz}, 0.06-$ $0.09 \mathrm{~Hz}, 0.09-0.12 \mathrm{~Hz}$ ) were confirmed by performing power spectrum density analysis. And significant measure channels were selected by performing one-way analyses of variance on three time periods around the start of movement. Force level was recognized by applying extreme learning machine (ELM). The corresponding precision rate was up to $78.7 \%$. The proposed identification method was not restricted to the existence of limbs or the strength of limb information. It was realized based on brain information recorded in a real movement environment; it is helpful to realize the desired force level of subjects and to provide a control command for rehabilitation training equipment.
\end{abstract}

\section{Introduction}

Recently, the number of people with motor dysfunction in lower limbs has been increasing rapidly because of natural disasters, injuries, accidents, diseases, and so on. In order to improve the training effect and the efficiency of recovery, patients' resistance training and active participation is very important and necessary. Thus, there is a great need to control rehabilitation device by patients' motion states of force level.

Generally, EMG and other biomechanical information were used to recognize motion states [1-3]. However, many patients lost important muscles or have little muscle functions, and thus EMG or biomechanical information was not enough to identify motion states for these patients. Recently, brain information obtained by using EEG and fMRI technology has been very popular around the world [4-6]. Dario Farina, et al. classified four actual cycling torque motion by EEG information, and the recognition rate was around 50.8\% [7]. DongEun Kim, et al. also applied EEG to classify the three grades $(25 \%, 50 \%, 75 \%)$ of hand grip MVC (Maximum Voluntary Contraction), and the result was about $52.03 \%$ for left hand and $77.7 \%$ for right hand [8]. However, the above research had strict restrictions on testing environment and subjects. By contrast, functional near infrared spectroscopy (fNIRS) is a suitable tool to record brain information. Baolei $\mathrm{Xu}$, et al. classified the speed and force clench imagination by support vector machine (SVM), the accuracy results was 72\% [9]. Yunfa $\mathrm{Fu}$, et al. combined the features of NIRS and EEG to recognize three levels of imagined force (at 20/50/80 \% MVGF (maximum voluntary grip force)) and speed (at $0.5 / 1 / 2 \mathrm{~Hz}$ ) of hand clenching by SVM. The average classification accuracy was $72 \%$ [10]. Xuxian Yin, et al. used concentration changes of oxy in the left hemisphere as input features to classify the imagined hand force and speed levels, and the recognition rate was around $75 \%$ [11]. However, similar research to identify motion states of lower limbs is very few. Studies [12-13] identified spontaneous motion states of lower limbs by using fNIRS. It confirmed the feasibility and advantage of fNIRS technology, especially for recognizing spontaneous motion states.

It is very important for patients to train the strength of lower limbs during the process of rehabilitation training. Moreover, controlling the resistance of lower limbs' movement by patients spontaneously is also necessary for promoting recovery. Therefore, the paper proposed a method to recognize the indented resistance of lower limbs' movement by applying fNIRS technology. Pedaling movement in three-class force 
levels was performed, and motion was controlled by the subjects themselves. Since Zengyong Li, et al [14] observed the meaningful features from prefrontal lobe in subjects at risk of stroke by spectral analysis, features in both the time-domain and the frequency-domain were considered in the paper. In addition, in order to reduce regional location error of individuals, information in the channels of interest rather than regions were analyzed. It was supposed that time-frequency features in the channels were preferable for recognizing motion states of spontaneous movements.

\section{Experiments}

\subsection{Subjects}

Ten right-handed healthy participants (10 male, age: $21 \pm 2$ years old) at Soochow University participated in riding bicycle experiment. None of them had a history of neurologic, psychiatric or other related disease. All the participants were informed of the test procedure before the experiment.

\subsection{Instruments}

A FORIE-3000 optical topography system (Shimadzu Corporation, Kyoto, Japan) [15] was used to measure concentration changes of oxygenated hemoglobin (oxy), deoxygenated hemoglobin (deoxy), and total hemoglobin (total). The distance between each pair of detector and emitter was $3 \mathrm{~cm}$. The sampling period of hemoglobin signals was $0.13 \mathrm{~s}$. An experimental diagram of riding bicycle is shown in Fig. 1 (a). Pedaling force was recorded by the rehabilitation bicycle device.

One set of $3 \times 5$ parietal flash holder was applied to fix emitters and detectors. In the experiment, 15 probes (7 detectors and 8 emitters) were used to collect cerebral activation data in a $3 \times 5$ area (Fig. 1 (b)). Based on the international 10-20 system [16-17], the probe holder was placed on the top of the head, and detector 7 was placed in the straight ahead of $\mathrm{Cz}$ portion with a distance of 3 cm. 22 channels are presented with digital numbers in Fig. 1 (b). The motor-related regions of interest included prefrontal cortices (PFC: channels 1, 2, 3, 4, 5, 6 and 7), supplementary motor areas (SMA: channels 14, 16, 17, 19, 21 and 22), premotor cortices (PMCL: channels 13 and 18 in the left hemisphere; PMCR: channels 15 and 20 in the right hemisphere). These regions have been proved to play an important role in controlling force [18]. The frontal eye cortices (FEC: channels 8, 9, 10, 11 and 12) was not the region of interest in our study.

\subsection{Motion task}

Pedaling task was performed in three levels of force (low resistance: $0 \mathrm{~N}$, medium resistance: $30 \mathrm{~N}$, and high resistance: $50 \mathrm{~N}$ ). Fig. 1 (c) illustrates the temporal sequence of the task design. Force movement task were first executed after a pre-rest period, and there was a rest between every two force tasks. As for each motion state, the task were performed two times. The start and end times of each task, as well as the time duration of each rest, were controlled by the subjects spontaneously. But the subjects were informed in advance to rest enough with a period not less than 25 seconds. The subjects were familiar with this time interval by exercising $2-3$ times before the experiment. During the experiment, the start and end of each task were recorded with a mark by an experiment operator. The hemoglobin concentration of the subjects was recorded simultaneously in the whole process of the test.

\section{Analysis Methods}

The concentration changes in the difference between oxy and deoxy, and that in the deoxy were analyzed. And the information in time-domain and frequency-domain were both analyzed for extracting hemoglobin features.

Firstly, power spectrum density analysis was carried out to observe principal frequency ranges of the data in different force levels. And based on spectral distribution characteristics, Chebyshev filtering method was used for performing band- pass filter.

Secondly, in order to avoid negative effects caused by bad data in baselines during the whole experiment, the change rate of oxy and deoxy (oxy rate and deoxy rate) in each concerned channel were calculated. The interval number of five was selected to calculate change rate. And as for each channel, the deoxy_rate and the difference between the means of oxy_rate and deoxy_rate (oxy_rate - deoxy_rate) were both calculated.
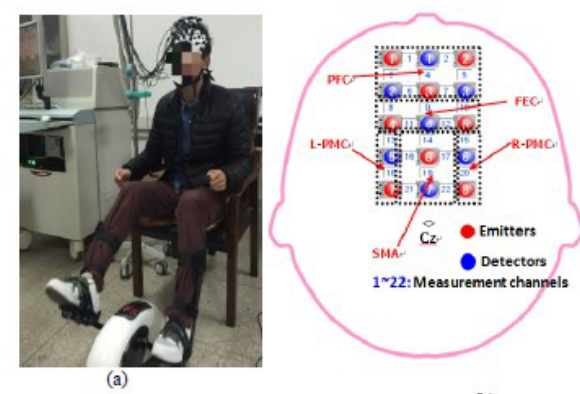

(b)

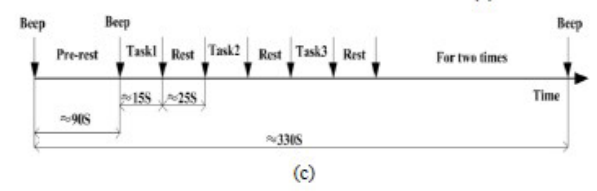

Figure. 1. (a) The experimental setup. (b) Distribution of the detected channels in motor-related region.(c) The task design. Task1: pedalling task with low force. Task2: pedalling task with medium force. Task3: pedalling task with high force.

Thirdly, to confirm the difference of frequency bands among the three force levels, average "oxy_ratedeoxy_rate" in all the channels were carried out for each sampling point. One ANOVA1 analysis on different frequency bands with the average values of "oxy_ratedeoxy_rate" in the period of T3 (nine points after the start mark were recorded as T3) was performed. Statistical significance of the task was set at $\mathrm{P}<0.05$.

Fourthly, to confirm the important channels in the time domain, as for each channel (exclude the channels of FEC), mean values of nine points before and after the motion start mark were recorded as $\mathrm{T} 2$ and $\mathrm{T} 3$, and 
mean value of nine points in front of $\mathrm{T} 2$ was recorded as T1. A one-way analysis of variance (ANOVA1) on 'time (T1, T2, T3)' was performed for each channel. The Fisher least significant difference test was used as a post hoc test. Statistical significance of the task was set at $\mathrm{P}<0.01$. Since the head size of subjects were not the same, the channels in the adjacent position of two regions may have some kind of positioning error. And the channels in each region may have opposite channel information. That is why the information in each channel rather than the average hemoglobin in each region was analyzed in our research.

At last, ELM recognition algorithm was adopted for distinguishing force levels. The algorithm solved the major bottleneck of feed-forward neural networks in learning speed, and provided a good performance at extremely fast learning speed [19]. In addition, ELM algorithm is easily implemented and less sensitive to specified parameters [20]. In this study, the number of hidden neurons in ELM was selected from100 to 2000, the step of 50 was selected.

\section{Results}

According to the feedback of investigation report written by subjects and the preliminary observation of data, all the subjects' data were chosen for analysis.

Based on power spectrum density analysis, the frequency corresponding to the power spectrum's peak of the concerned channels were presented in Fig. 2.

It is found that the main frequency bands in different force levels were different. In level of $0 \mathrm{~N}$, the main frequency in each channel was almost below $0.03 \mathrm{~Hz}$. In the level of $50 \mathrm{~N}$, the main frequency in most channels was between $0.03 \mathrm{~Hz}$ and $0.06 \mathrm{~Hz}$. The results were consistent with the conclusion of the paper written by $\mathrm{Z}$. $\mathrm{Li}$ [21]. He has proved that the oscillation in interval between $0.02-0.08 \mathrm{~Hz}$ was considered to reflect the neurogenic activity in the vessel wall. In order to avoid losing some useful information, the analyzed frequency range was increased to $0.01-0.12 \mathrm{~Hz}$. Band-pass filter with four bands ( I: $0.01-0.03 \mathrm{~Hz}$, II: $0.03-0.06 \mathrm{~Hz}$, III: $0.06-0.09 \mathrm{~Hz}$, IV: $0.09-0.12 \mathrm{~Hz}$ ) was performed respectively. By performing one-way analysis on the four frequency bands with the average "oxy_ratedeoxy_rate" in T3, it is found that the main frequency bands in different force levels were significantly different. The results are given in Fig. 3. During the low force motion, band IV was lower than others $(\mathrm{P}=0.000)$ and the band I was larger than band II $(\mathrm{P}=0.002)$. As for the medium force, there are no significant characters. At the high force, band I was lower than band II ( $\mathrm{P}=0.01)$, and band III $(\mathrm{P}=0.016)$ and IV was larger than others $(\mathrm{P}=0.000)$.

To confirm the important channels during each task with the mean values of T1, T2 and T3. When the mean value in T3 was obviously larger or smaller than those in both $\mathrm{T} 1$ and $\mathrm{T} 2$, and simultaneously, and the two means in $\mathrm{T} 1$ and $\mathrm{T} 2$ have no significant difference, the channel was selected as an important one. The deoxy and "oxy_rate-deoxy_rate" were both used for selecting important channels.

0N: as shown in Fig. 4, "oxy_rate-deoxy_rate" of channel 11 in T3 was significantly lower than those both in $\mathrm{T} 2$ and $\mathrm{T} 1(\mathrm{P}=0.001)$. And the values of deoxy in T3 were obviously different from those both in $\mathrm{T} 2$ and $\mathrm{T} 1$ for the channels $2(\mathrm{P}=0.000)$ and $3(\mathrm{P}=0.000)$. Thus, the channels of 2,3 , and 11 were considered as important channels for recognizing low force level.

$30 \mathrm{~N}$ : As for the medium force level (Fig. 5), there was not any important channel being selected with the values of "oxy_rate-deoxy_rate". As for deoxy, the values of channel $8(\mathrm{P}=0.000)$ in $\mathrm{T} 3$ was greatly larger than those in $\mathrm{T} 1$ and $\mathrm{T} 2$.

$50 \mathrm{~N}$ : As for the high force level (Fig. 6), "oxy ratedeoxy_rate" of channels $11,12(\mathrm{P}=0.000), 13(\mathrm{P}=0.000)$, $17(\mathrm{P}=0.000), 21 \quad(\mathrm{P}=0.004)$ in $\mathrm{T} 3$ was obviously larger than those in the T1 and T2. And deoxy of channel 2 $(\mathrm{P}=0.000), 4(\mathrm{P}=0.003)$ in $\mathrm{T} 3$ was also selected by the same method. The above channels were useful for recognizing high force level.

Overall, all the important channels (eight channels: $2,3,4,8,11,12,13,17,21)$ in the three force levels were chosen for reorganizing force levels, as summarized in TABLE I. And the four frequency bands were all selected. For each important channel, the average values in T3 were carried out for each frequency band. Thus, there was 36 feature vector (4 frequency bands $\times$ ("oxy_rate-deoxy_rate" in 11,12,13,17,21 + "deoxy_rate" in the channels of 2,3,4,8) ). The format of training set and test set were listed in TABLE II. As for ten subjects, the 16 rows represented the characters of eight training subjects ( 8 subjects $\times 2$ times) in each force level. rows of the test set were corresponding results of other two subjects $(2$ subjects $\times 2$ times $\times 3$ force levels).

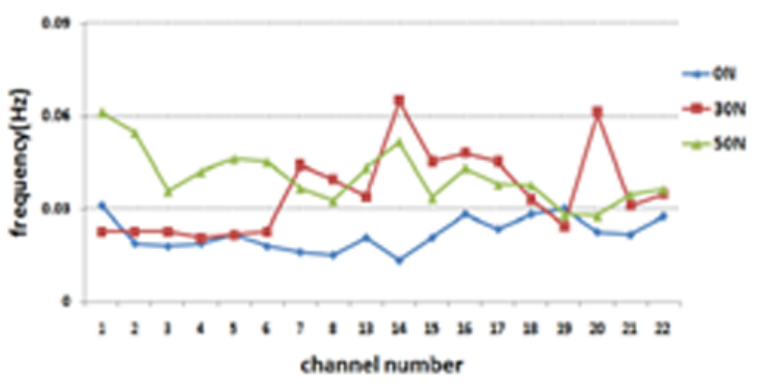

Figure. 2. The power spectrum's peak of the concerned channels (except for channels in FEC) in different force levels.

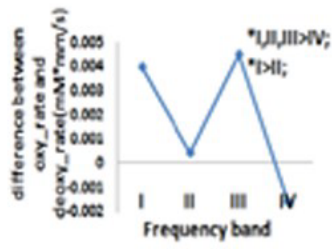

(a)

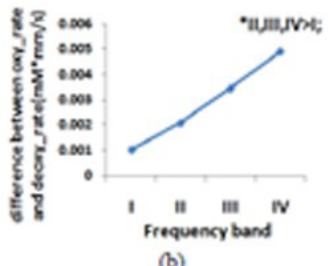

Figure. 3. (a) Characters of frequency bands in low force level. (b) Characters of frequency bands in low force level. Medium force level was not found significant characters. I, II, III, and IV represent the frequency band of $0.01-0.03 \mathrm{~Hz}, 0.03-0.06 \mathrm{~Hz}$, $0.06-0.09 \mathrm{~Hz}$, and $0.09-0.12 \mathrm{~Hz}$, respectively. Significant difference level: ${ }^{*} \mathrm{p}<0.05$. 

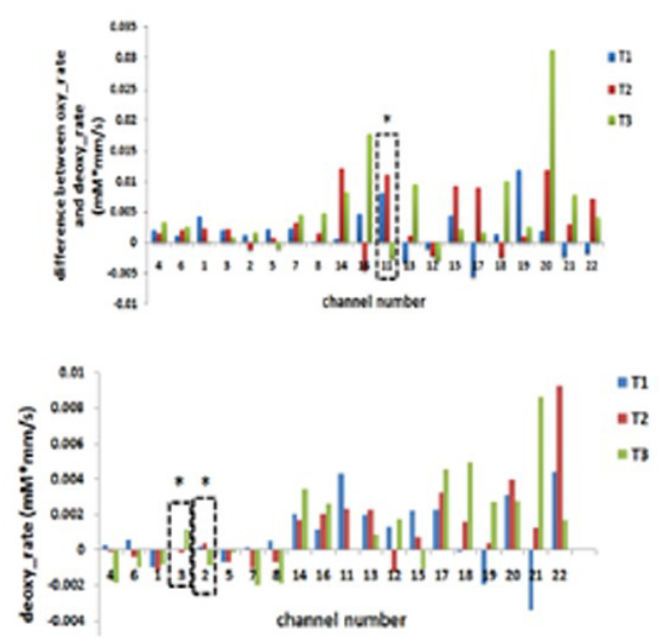

Figure. 4. $0 \mathrm{~N}$ force level: (a) the average the difference between oxy and deoxy in the period of T1, T2 and T3. (b) the average deoxy in the period of T1, T2 and T3. When T3 was significantly larger or lower than $\mathrm{T} 1$ and $\mathrm{T} 2$, and $\mathrm{T} 1$ and $\mathrm{T} 2$ have no significant difference, the channel were marked with black dotted box. Significant difference level: ${ }^{*} \mathrm{P}<0.01$.

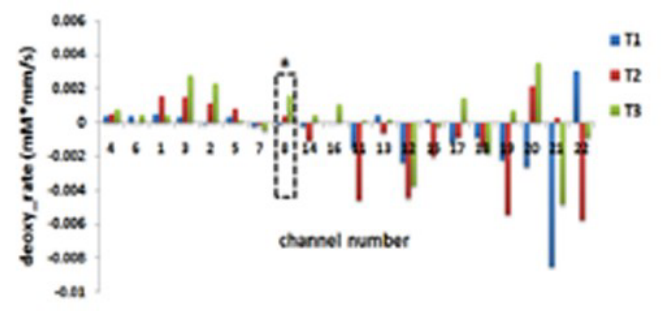

Figure. 5. 30N force level: the average deoxy in the period of $\mathrm{T} 1, \mathrm{~T} 2$ and $\mathrm{T} 3$. When $\mathrm{T} 3$ was significantly larger or lower than $\mathrm{T} 1$ and $\mathrm{T} 2$, and $\mathrm{T} 1$ and $\mathrm{T} 2$ have no significant difference, the channel were marked with black dotted box. Significant difference level: ${ }^{*} \mathrm{P}<0.01$.

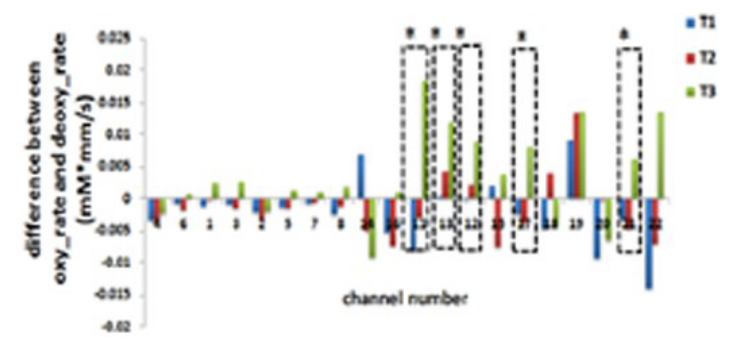

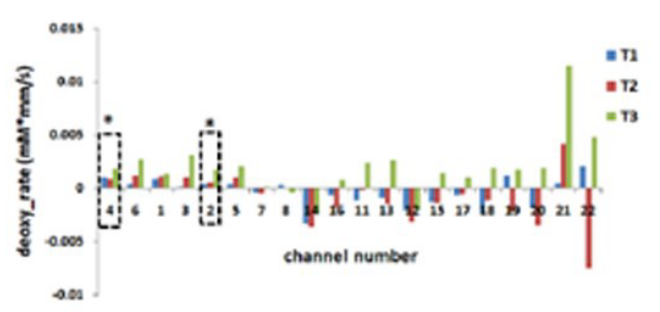

Figure. $6.50 \mathrm{~N}$ force level: (a) the average the difference between oxy and deoxy in the period of T1, T2 and T3. (b) the average deoxy in the period of T1, T2 and T3. When T3 was significantly larger or lower than $\mathrm{T} 1$ and $\mathrm{T} 2$, and $\mathrm{T} 1$ and $\mathrm{T} 2$ have no significant difference, the channel were marked with black dotted box. Significant difference level: ${ }^{*} \mathrm{P}<0.01$.

Table1. Channels of Interest during EachForce Level

\begin{tabular}{|c|c|c|}
\hline \multirow{2}{*}{} & $\begin{array}{c}\text { The channel number of interest } \\
\text { channels (deoxy/the difference of }\end{array}$ \\
\cline { 2 - 3 } & Increasing & Decreasing \\
\hline Low force & $3 /$ none & $2 / 11$ \\
\hline Medium force & $8 /$ none & none \\
\hline High force & $2,4 /$ & none \\
\hline
\end{tabular}

Table2. ELM: Training Data and Testing data

\begin{tabular}{|c|c|}
\hline task & $\begin{array}{c}\text { Characters (four frequency bands and eight } \\
\text { channels) }\end{array}$ \\
\hline Low Force & 16 rows $\times 36$ characters ${ }^{\mathrm{a}}$ \\
\hline $\begin{array}{c}\text { Medium } \\
\text { speed }\end{array}$ & 16 rows $\times 36$ characters $^{\mathrm{b}}$ \\
\hline High Force & 16 rows $\times 36$ characters $^{\mathrm{c}}$ \\
\hline Testing & 12 rows $^{\mathrm{c}} \times 36$ characters $^{\mathrm{d}}$ \\
\hline
\end{tabular}

a. Training data in low force motion of eight subjects.

b. Training data in medium force motion of eight subjects.

c. Training data in highforce motion of eight subjects.

d. Testing data in low force motion of two subjects.

The best number of hidden neurons in ELM was 150 in this study. The mean of identification rates were $78.9 \%, 80 \%$, and $77.3 \%$ respectively for the $0 \mathrm{~N}, 30 \mathrm{~N}$ and $50 \mathrm{~N}$ force levels. The average precision rate was up to $78.7 \%$.

\section{Discussion}

The paper introduced a method to identify force level of pedaling based on cerebral hemoglobin information. The information in time-domain and frequency-domain were analyzed to extract features. Additionally, information in the channels of interest rather than regions were analyzed to reduce regional location error of individuals. ELM method was applied for classification with a recognition rate $78.7 \%$ being achieved. The proposed identification method is favorable to identify motion states of lower limbs in a real environment, and further to improve the effect of rehabilitation training.

Currently, there are many methods to select optimal features for pattern recognition. The paper [22] 
investigated different time window combinations during the task period. The optimal time was selected to achieve a better accuracy. The identification rate of right and left motor imagery was $75.6 \%$. Vikas, et al. presented a classification-guided (wrapper) method for extracting NIRS feature in time-domain, and then to classify left and right hand movements. The identification rate can achieve 100\% [23]. Based on univariate and multivariate ranking and sequential forward and backward selection, four different wrapper methods and three different classifiers (k-Nearest neighbor, Bayes, and Support Vector Machines) were studied. The above researches considered features in time-domain only. In our study, the information in timedomain and frequency-domain were both analyzed, and the features in frequency-domain provided characteristic differences additionally. Parameters of nine important channels in the four independent frequency bands were selected as input features. And a relatively good recognition rate of $78.7 \%$ was achieved for spontaneous motion states without evoked stimulation.

At present, the researches on identifying motion states of lower limbs are very few. The study [24] combined EEG and NIRS for some actual motion (right/left arm and hand task) identification. Their accuracy of identifying right and left motion was $72.2 \%$, and the accuracy of classifying arm and hand tasks was $79.9 \%$. The study [25] used EEG-fNIRS feature for decoding motor imagery of both force and speed of hand clenching, it achieved an accuracy of $89 \% \pm 2 \%$. In comparison, by using fNIRS technology alone, our proposed method identified force levels of lower limb with an accuracy of $78.7 \%$. This is meaningful for controlling rehabilitation device relatively simple. Overall, the current result lays a foundation of realizing autonomous control of rehabilitation training equipment and it may be great benefit for the research on rehabilitation robots. This will be more preferable to motivate patients' active participation and enhance training effect.

\section{Acknowledgment}

This work was supported by a grant from the national high technology research and development program (863 Program: 2015AA042301), China Postdoctoral Science Foundation (2015M580461).

\section{References}

[1] S. Lee, "Power assist control for walking aid with HAL-3 based on EMG and impedance adjustment around knee joint. "in Proc. IEEE/RSJ Int. Conf. Intelligent Robots and Systems (IROS 2002), Lausanne, Switzerland, pp. 1499-1504, 30 Sep.-4 Oct, 2002.

[2] T. Kagawa, "A human interface for stride control on a wearable robot. "in Proc. IEEE/RSJ Int. Conf. Intelligent Robots Systems (IROS 2009), St. Louis, MO, USA, pp. 4067-4072, 11-15 Oct., 2009.

[3] T. Hayashi., "Control method of robot suit HAL working as operator's muscle using biological and dynamical information."in Proc. IEEE/RSJ Int. Conf. Intelligent Robots and Systems (IROS 2005), pp. 3063-3468, 2-6 Aug, 2005.

[4] Farwell, "Talking off the top of your head: toward a mental prosthesis utilizing event-related brain potentials."Electroencephalography and clinical Neurophysiology 70(6), pp.510-523, 1988.

[5] Weiskopf, Nikolaus, et al. "Principles of a braincomputer interface (BCI) based on real-time functional magnetic resonance imaging (fMRI)." IEEE transactions on biomedical engineering 51(6), pp. 966-970, 2004.

[6] Serruya, "Brain-machine interface: Instant neural control of a movement signal." Nature 416(6877), pp.141-142, 2002.

[7] Farina, D., Optimization of wavelets for classification of movement-related cortical potentials generated by variation of force-related parameters. Journal of neuroscience methods, 162(1), pp.357-363,2007.

[8] Kim, Dong-Eun, et al. "EEG analysis following change in hand grip force level for BCI based robot arm force control." Journal of Korean Institute of Intelligent Systems 23.2 (2013): 172-177.

[9] Xu, B., Fu, Y., Miao, L., Wang, Z., \& Li, H. (2011, December). Classification of fNIRS data using wavelets and support vector machine during speed and force imagination. In Robotics and Biomimetics (ROBIO), 2011 IEEE International Conference on (pp. 1224-1229). IEEE.

[10] FU Yun Fa. "Recognition of Actual Grip Force Movement Modes Based on Movement-related Cortical Potentials." Acta Automatica Sinica6,2013, pp:1045-1057.

[11] Yin, X., Xu, B., Jiang, C., Fu, Y., Wang, Z., Li, H., \& Shi, G.. Classification of Hemodynamic Responses Associated With Force and Speed Imagery for a Brain-Computer Interface. Journal of medical systems,39(5), pp.1-8,2015.

[12] Li, C. (2015). Identification of motion trend of lower limbs based on near-infrared spectroscopic technology. Advances in Mechanical Engineering, 7(3), 1687814015575979.

[13] Li, C., Li, J., Hu, H., Li, W., Ren, Z., \& Qu, W.. Adjustment of Intention to Start a Motion of Lower Limbs Based on Cerebral Hemoglobin Information. In Intelligent Human-Machine Systems and Cybernetics (IHMSC), 2015 7th International Conference on Vol. 1, pp. 568-573. IEEE, 2015, August.

[14] Li, Zengyong, "Spectral analysis of near-infrared spectroscopy signals measured from prefrontal lobe in subjects at risk for stroke." Medical physics39(4), pp.2179-2185, 2012.

[15] Shimadzu Corporation, Kyoto, Japan, Instruction manual for functional optical imaging system FOIRE-3000 series, Manual No. : M587-E005, Rev. B, Oct. 2010. 
[16] Okamoto, Three-dimensional probabilistic anatomical cranio-cerebral correlation via the international 10-20 system oriented for transcranial functional brain mapping. Neuroimage, 21(1),pp. 99-111,2004.

[17] D. Tsuzuki, "Virtual spatial registration of standalone fNIRS data to MNI space, " NeuroImage, vol. 34, pp. 1506-1518, 2007.

[18] N. Picard, "Imaging the premotor areas." Current opinion in neurobiology 11(6), pp.663-672,2001.

[19] Huang, G. B., Zhu, Q. Y., \& Siew, C. K.. Extreme learning machine: theory and applications. Neurocomputing, 70(1), pp.489501,2006.

[20] Huang, "Extreme learning machine for regression and multiclass classification." IEEE Transactions on Systems, Man, and Cybernetics, Part B (Cybernetics) 42(2), pp. 513-529, 2012.

[21] Li, Zengyong, et al. "Wavelet analysis of skin blood oscillations in persons with spinal cord injury and able-bodied subjects." Archives of physical medicine and rehabilitation 87.9 (2006): 1207-1212.

[22] Hong, "Classification of prefrontal and motor cortex signals for three-class fNIRSBCI." Neuroscience letters 587, pp, 87-92. 2015.

[23] Gottemukkula, "Classification-guided feature selection for NIRS-based BCI." International Ieee/embs Conference on Neural Engineering IEEE, 2011:72-75.

[24] Buccino, "Hybrid EEG-fNIRS Asynchronous Brain-Computer Interface for Multiple Motor Tasks." PloS one 11(1), pp.e0146610, 2016.

[25] Kai, Keng Ang, J. Yu, and C. Guan. "Extracting effective features from high density nirs-based BCI for assessing numerical cognition." 22.10,2012, pp:2233-2236.

[26] Zikai Yi, "statically stable walking gait planning of a kind of exoskeleton walking aid robot". Journal Of Mechanical Transmission (J), 40(1), pp. 48-53,2016. 